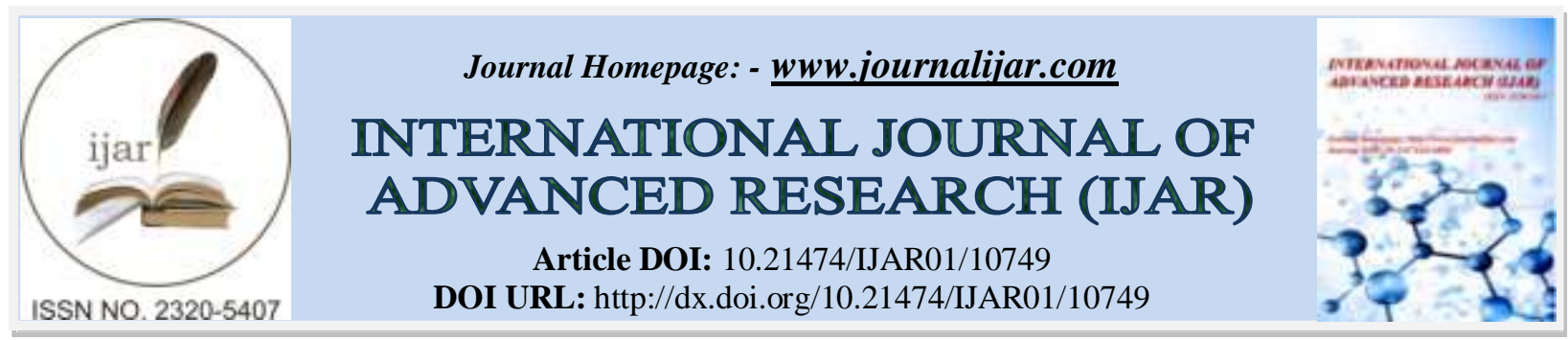

RESEARCH ARTICLE

\title{
PREVALENCE OF THYROID DYSFUNCTION IN PATIENTS OF CIRRHOSIS OF LIVER AND ITS CORRELATION WITH SEVERITY OF CIRRHOSIS
}

\author{
Dr. Ashish Kumar, Dr. Vivek Ahuja, Dr. Inderjit kaur, Dr. Vandna Pandov, Dr. Amritpal Singh and Dr. \\ RPS Sibia
}

Department of Medicine, Government Medical College Patiala. Punjab. India.

\section{Manuscript Info}

............................

Manuscript History

Received: 05 February 2020

Final Accepted: 07 March 2020

Published: April 2020

Key words:-

Cirrhosis, Thyroid Dysfunction/

Disorder

\section{Abstract}

Introduction: Abnormalities in thyroid hormones level have been observed commonly in patients of cirrhosis.

Aim: To determine the prevalence of thyroid dysfunction in liver Cirrhosis and its correlation with severity of cirrhosis

Materials and Methods: Early morning fasting serum thyroid stimulating hormone (TSH), serum thyroxine (T4) and serum triiodothyronine (T3) were measured by radioimmunoassay in 50 consecutive patients with liver cirrhosis (irrespective of etiology) who did not have prior history of thyroid disease. Patients with known thyroid disease, patients who had any previous thyroid surgery or exposure to radioactive iodine in past were excluded from this study. The age range of our study population was $27-80$ years with mean age being 47.82 years. Out of the 50 patients, 35 were males $(70 \%)$ and 15 were females $(30 \%)$ with male to female ratio of 2.33 . Twenty one patients had alcoholic liver disease, 20 had Hepatitis C, 5 had hepatitis $\mathrm{B}$ and 4 patients had cryptogenic cirrhosis. Severity of cirrhosis was categorized by using Child-Turcott-Pugh (CTP) criteria. On assessment of severity of cirrhosis 26 patients belonged to CTP A, 19 to CTP B and 5 to CTP C.

Results: Subclinical hypothyroidism was seen in 5 out of 50 patients $(10 \%)$ and hyperthyroidism was observed in 2 cases $(4 \%)$. Of the 5 patients having hypothyroidism, 2 belonged to CTP A (out of 26 i.e 7.69\%), 1 belonged to CTP B (out of 19 i.e $5.26 \%$ ), and 2 belonged to CTP C (out of 5 i.e 40\%). Among the patients with hypothyroidism, 3 (60\%) had ethanol related liver cirrhosis, 1 (20\%) had Hepatitis C whereas 1 had cryptogenic cirrhosis (20\%). On the other hand 3 out of 21 patients $(14.28 \%)$ with ethanol related liver cirrhosis, 1 out of 20 patients $(5 \%)$ with Hepatitis $\mathrm{C}$ related liver cirrhosis and 1 out of 4 patients $(25 \%)$ with cryptogenic cirrhosis had hypothyroidism. Two Patients with hyperthyroidism belonged to CTP A; one had cryptogenic cirrhosis and one has hepatitis $\mathrm{C}$.

Conclusion: High incidence of abnormalities in circulating thyroid hormone concentrations i.e hypothyroidism is noted especially in those with ethanol related liver cirrhosis and it is associated with more advanced liver disease $(\mathrm{p}=0.02)$

Corresponding Author:- Dr. Amritpal Singh

Address:- Department of Medicine, Government Medical College Patiala. Punjab. India. 

Abbreviations: CTP-Child Turcott Pugh, TSH- Thyroid Stimulating
Hormone

Copy Right, IJAR, 2020,. All rights reserved.

\section{Introduction:-}

Liver dysfunction has significant impact on the metabolism of thyroid hormones. Liver is involved in the conjugation and biliary excretion of thyroid hormones as well as extrathyroidal deiodination of thyroxine (T4) to triiodothyronine (T3) and to reverse T3 [1-4]. It also plays important role in the synthesis of proteins that bind thyroid hormones such as thyroxin binding globulin (TBG), prealbumin and albumin thereby influencing their serum levels. Similarly normal thyroid hormone levels are essential for the optimal functioning of hepatocytes [5]. Although patients of cirrhosis are mostly euthyroid clinically, many studies in the past have reported various abnormalities in the circulating thyroid hormones. The data from these studies is inconsistent and with variable findings [6-9]. There is paucity of data on the thyroid function in patients of cirrhosis from this region. Therefore we carried out this study to assess the prevalence of thyroid dysfunction in patients of liver cirrhosis and its association with the severity of cirrhosis.

\section{Material and Methods:-}

It was a cross sectional observational study carried out between the period of April and September of 2018 on patients reporting to inpatient and outpatient departments of Medicine of our instititute. Early morning fasting serum thyroid stimulating hormone (TSH), serum thyroxine (T4) and serum triiodothyronine (T3) were measured by radioimmunoassay in 50 consecutive patients with liver cirrhosis who did not have prior history of thyroid disease. All the patients included in the study had established diagnosis of liver cirrhosis based on clinical, biochemical and radiological parameters. Clearance was taken from ethical committee before embarking upon this study. Informed written consent was obtained from every patient included in the study.

\section{Inclusion criteria:}

All the patients of liver cirrhosis (irrespective of etiology), attending the Medicine outpatient department (OPD) of our institute were included in this study.

\section{Exclusion criteria:}

Patients of liver cirrhosis with known thyroid disease, thyroid surgery or exposure to radioactive iodine in past were excluded from the study.

The age range of our study population was 27-80 years with mean age being 47.82 years. Out of the 50 patients, 35 were males $(70 \%)$ and 15 were females $(30 \%)$ with male to female ratio of 2.33 . Twenty one (42\%) patients had alcoholic liver disease, $20(40 \%)$ had Hepatitis C, $5(10 \%)$ had hepatitis B and $4(8 \%)$ patients had cryptogenic cirrhosis (Figure 1). Severity of cirrhosis was categorized by using Child-Turcott-Pugh (CTP) criteria. On assessment of severity of cirrhosis $26(52 \%)$ patients belonged to CTP A, (38\%) to CTP B and (10\%) to CTP C (figure 2).

\section{Results:-}

All the patients included in the study were clinically euthyroid. Biochemical hypothyroidism was seen in 5 out of 50 patients (10\%) while hyperthyroidism was observed in 2 cases (4\%) (Figure 3). Among the patients with biochemical hypothyroidism (elevated TSH) only one patient had low T3 while all had normal T3 and T4 levels. Of the 5 patients with hypothyroidism, 2 belonged to CTP A (out of 26 i.e $7.69 \%$ ), 1 belonged to CTP B (out of 19 i.e $5.26 \%$ ), and 2 belonged to CTP C (out of 5 i.e $40 \%$ ).

Among the patients with hypothyroidism, $3(60 \%)$ had ethanol related liver cirrhosis, $1(20 \%)$ had Hepatitis $\mathrm{C}$ whereas I had cryptogenic cirrhosis (20\%) (Figure 4). On the other hand 3 out of 21 patients (14.28\%) with ethanol related liver cirrhosis, 1 out of 20 patients (5\%) with Hepatitis C related liver cirrhosis and 1 out of 4 patients (25\%) with cryptogenic cirrhosis had hypothyroidism.

Two of our patients had biochemical hyperthyroidism with low TSH, high T3 and normal T4. Both patients (females) belonged to CTP A; one had hepatitis C related cirrhosis while other one had cryptogenic cirrhosis (Figure: 3). Therefore all the patients with biochemical abnormalities in thyroid hormones had normal T4 levels 


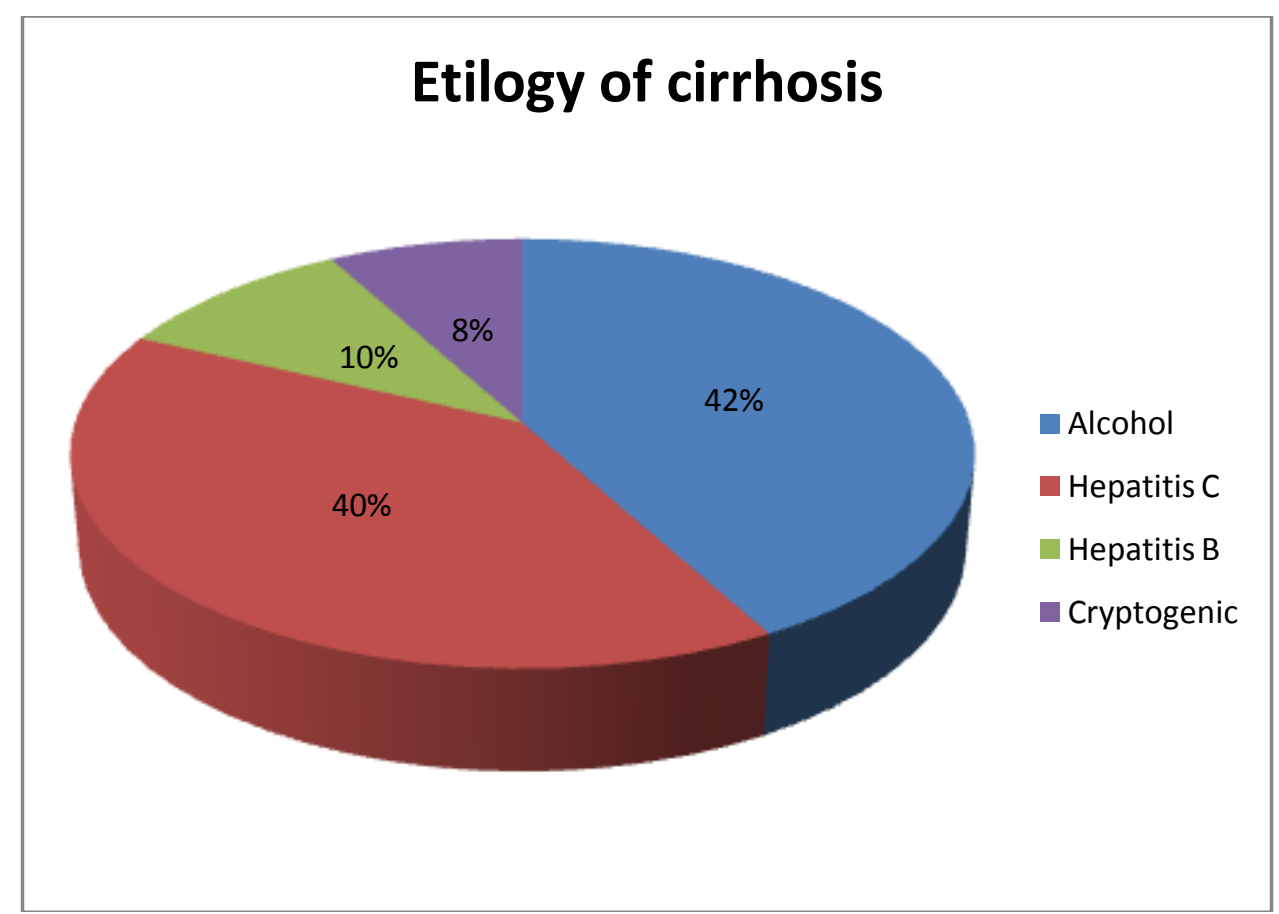

Figure 1:- Etiology of cirrhosis.

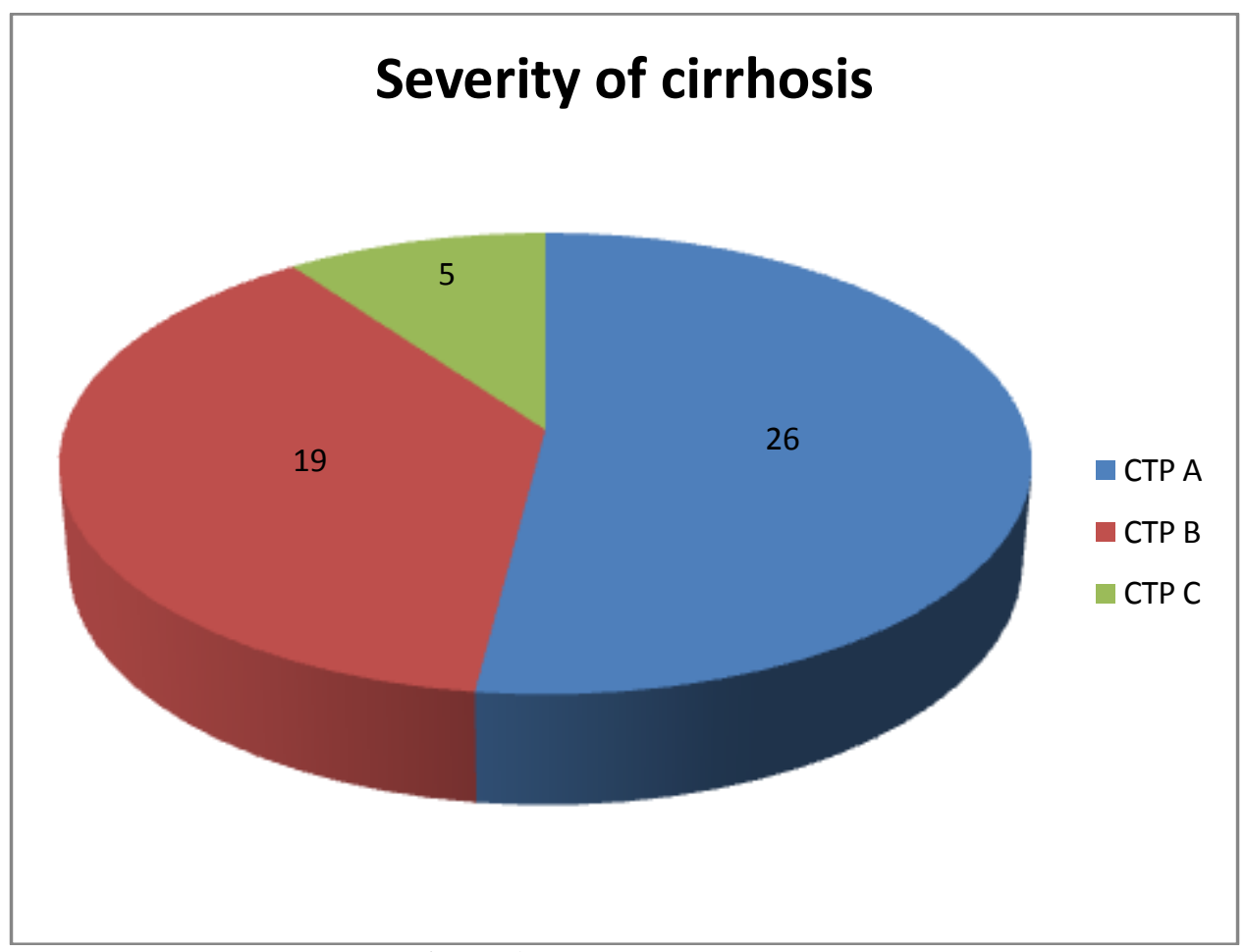

Figure 2:- Severity of cirrhosis. 


\section{Prevalance of thyroid dysfunction in patients of cirrhosis}

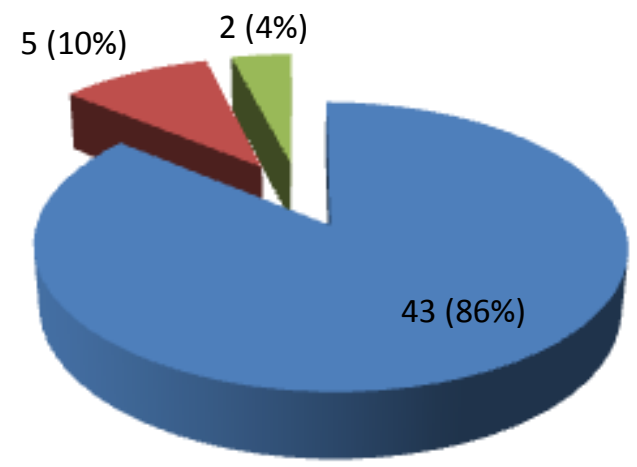

Euthyroid

Hypothyroidism

- Hyperthyroidism

Figure 3:- Prevalence of thyroid dysfunction in patients of cirrhosis.

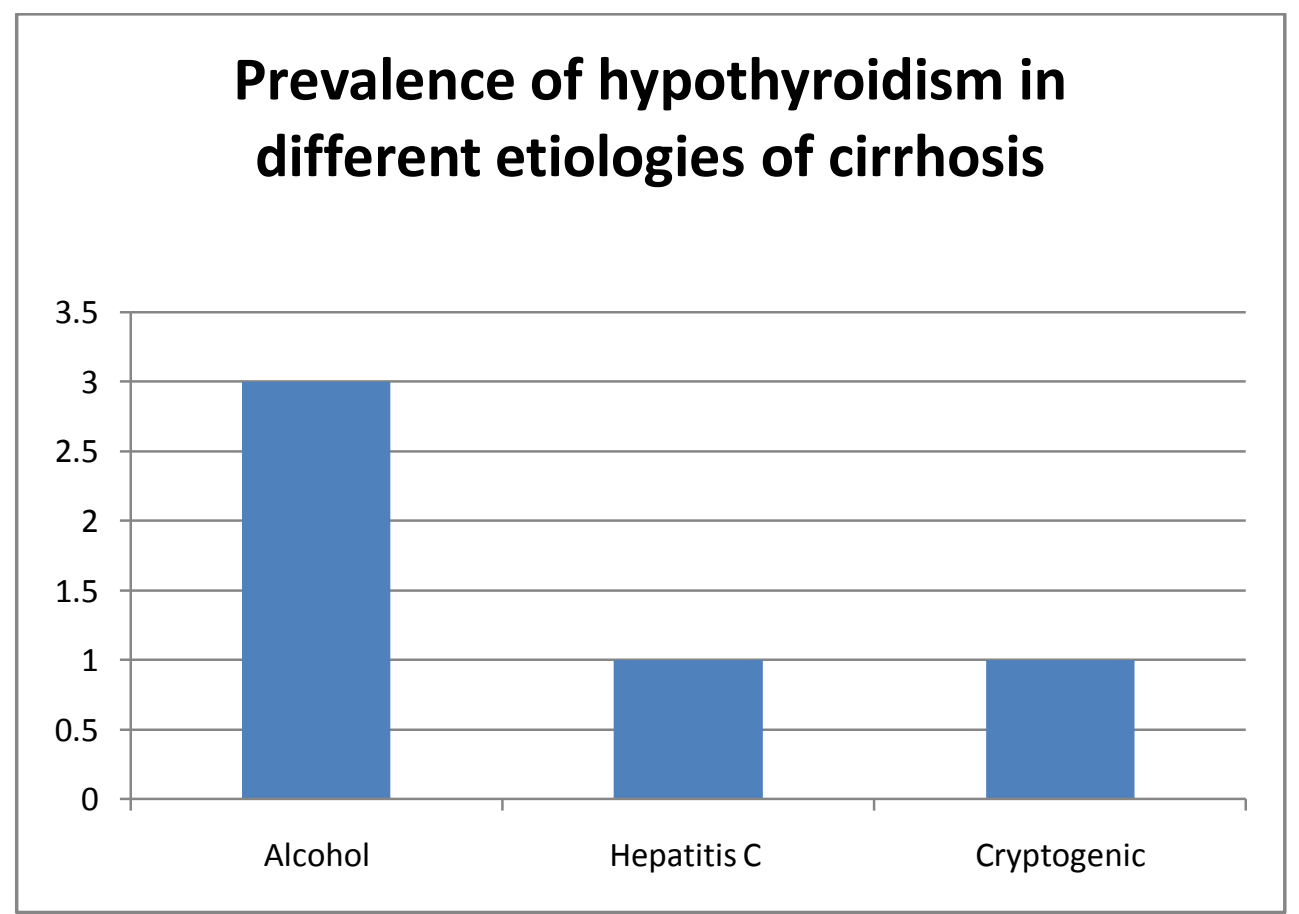

Figure 4:- Prevalence of hypothyroidism in different etiologies of cirrhosis.

\section{Discussion:-}

Thyroid dysfunction has been reported in several chronic diseases including cirrhosis of liver. In our study $10 \%$ of the patients with cirrhosis had biochemical evidence of hypothyroidism while $4 \%$ had biochemical hyperthyroidism. Unlike many studies in past [1-4] our patients showed high TSH with normal T3 (except in one patient) and T4. Previously studies have reported sick euthyroid syndrome also known as low T3 syndrome which is characterized by reduction of extra-thyroidal T4-to-T3 conversion and, consequently, a low total T3, low free T3, an elevated rT3 (reverse T3), normal/low total T4 and normal/high free T4 [4-9]. In a small study Borzio et al reported significantly low T3, FT3, T3/thyroxine binding globulin and T4/thyroxine binding globulin ratios and 
TSH, while elevated fT4, thyroxine binding globulin [4]. Joeimon et el from India demonstrated high incidence of subclinical hypothyroidism (21.6\%) characterized by elevated TSH and low T3 in 50\% of the patients with hypothyroidism. Remaining $50 \%$ of hypothyroid patients had normal FT3, FT4 raised TSH [7]. No study has shown hyperthyroidism in patients of cirrhosis seen in our study.

The relationship between severity of cirrhosis and thyroid hormones has been suggested in many studies in the past $[4,9,10]$. In our study high proportion of patients with severe cirrhosis (CTP C) had evidence of hypothyroidism. But this number was too small to draw any inference. Some studies have demonstrated an inverse correlation between serum T3 concentrations and the severity of liver dysfunction. Thyroid function has been evaluated as a marker of prognosis of liver disease and the thyroid function abnormalities usually are reversed following liver function improvement and liver transplant [11,12]. Therefore relationship between cirrhosis and thyroid hormones remains inconclusive.

\section{Conclusion:-}

1. Significant number of patients with cirrhosis of liver shows abnormalities in circulating thyroid hormone concentrations who are clinically euthyroid.

2. There is a suggestion of relationship between severity of cirrhosis and abnormalities in the thyroid hormone levels.

3. Further studies are needed to confirm our findings and to find out if treatment of thyroid dysfunction has any bearing on mortality and morbidity of cirrhosis.

\section{Conflict of interest:}

Authors have no conflict of interest to declare.

\section{Bibliography:-}

1. Chopra IJ, Solomon DH, Chopra U, Young RT, Chuateco GN. Alterations in circulating thyroid hormones in hepatic cirrhosis; evidence for euthyroidism despite subnormal serum triiodothyronine. J Clin Endocrinol Metab 1974; 39: 501-11.

2. Nomura S, Pittman CS, Chambers JB, Buck MW,Shimizu T. Reduced peripheral conversion of thyroxine to triiodothyronine in patients with hepatic cirrhosis. J Clin Invest 1975; 56: 643-52. (INTRODUCTION \& PERIPHERAL DEIODINATION)

3. Chopra IJ, Solomon DH, Hepner GW, Morgestein AA. Misleadingly low free thyroxine index and usefulnesses. Ann Intern Med 1979; 90: 905-12.

4. M BORZIO, R CALDARA, F BORZIO, V PIEPOLI, P RAMPINI, AND C FERRARI. Thyroid function tests in chronic liver disease: evidence for multiple abnormalities despite clinical euthyroidism. Gut, 1983, 24, 631636

5. R. Malik, H. Hodgson, The relationship between the thyroid gland and the liver, QJM: An International Journal of Medicine, 95(9);2002;559-69,

6. Seehofer D, Steinmueller T, Graef KJ, Rayes N, Wiegand W, Tullius SG, Settmacher U, Neuhaus P. Pituitary Function Test and Endocrine Status in Patient with Cirrhosis of the Liver before and after Hepatic Transplantation. Ann Transplant. 2002;7(2):32-7.

7. Joeimon J L, Mohanraj K, Karthikeyan R, Rajkumar Solomon T, Aravind A, Caroline Selvi K, Balamurali R, Ramkumar G, Muthukumuran K,Vaishnavipriyaa C,Kavitha S,Anand A. Thyroid Dysfunction in Patients with Liver Cirrhosis. Volume 16, Issue 4 Ver. VIII (April. 2017), PP 18-22 Journal of Dental and Medical Sciences.

8. Green JR. Thyroid function in chronic liver disease Z Gastroenterol. 1979 Jul;17(7):447-51

9. Shimada T, Higashi K, Umeda T, Sato T. Thyroid functions in patients with various chronic liver diseases. EndocrinolJpn. 1988 Jun; 35(3):357-69.

10. Shakoor S, Kaneez FS, Iftikhar U. Free $\mathrm{T} 3$ as a Reliable Indicator of Thyroid Dysfunction in Cirrhosis. Iamure.2012;1(1).

11. Israel Y, Walfish PG, Orrego H, Blake J, Kalant H.Thyroid hormones in alcoholic liver disease: effect of treatment with 6-propylthiouracil. Gastroenterology 1979; 76: 116-22.

12. Karla Rocha PENTEADO, Júlio Cezar Uili COELHO, Mônica Beatriz PAROLIN, Jorge Eduardo Fouto MATIAS, Alexandre Coutinho Teixeira de FREITAS. The influence of end-stage liver disease and liver transplantation on thyroid hormones. Arq. Gastroenterol. vol.52 no.2 São Paulo April./June 2015. 